\title{
Die Ärztegesellschaft des Kantons Jura
}

Remo Osterwalder

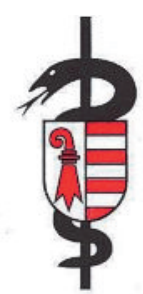

SOCIÉTÉ MÉDICALE DU CANTON DU JURA

Korrespondenz: Dr. med. Remo Osterwalder Präsident der SMCJU Rue du 24-Septembre 7 CH-2800 Delsberg Tel. 0324234040 Fax 0324234044

\section{Historisches}

Die Gesellschaft ist in ihrer heutigen Form und Struktur eine der jüngsten, denn sie geht mit der Gründung und Verselbständigung des Kantons Jura im Jahr 1979 einher. Ursprünglich war sie zusammen mit den Kollegen des südlichen Berner Juras, der heute noch mit einer eigenständigen Sektion in der Berner Ärztegesellschaft besteht. Mit den Kollegen des südlichen Juras besteht eine gute Beziehung, was jedes Jahr mit einer gemeinsamen Generalversammlung Ende Jahr gefördert wird. Die Gesellschaft hat heute 152 Mitglieder, wovon rund 100 in eigenständigen oder GruppenPraxen praktizieren. Davon sind 50 Inhaber des Titels Allgemein- oder Innere Medizin.

\section{Probleme}

Als Grenzkanton und gleichzeitig nördlichster französischsprachiger Kanton sind wir mit speziellen Problemen konfrontiert. Diese sind insbesondere in der Grundversorgung am deutlichsten. Für viele Romands sind wir weitab von den grossen frankophonen Zentren wie Lausanne und Genf. Intensive Zusammenarbeit besteht vor allem mit dem Kanton Neuenburg (z. B. Ethikkommission, Pandemie). Weiter ist auch die Zusammenarbeit mit der Nordwestschweiz, insbesondere mit Basel, naheliegend, da deren universitäres Zentrum für uns am nächsten liegt.

Die Probleme der Grundversorgung und insbesondere die Organisation des Notfalldienstes machte eine Anhebung der Alterslimite auf 65 Jahre nötig, und zurzeit sind wir in Evaluation eines Projektes zur Zusammenlegung, respektive Zusammenarbeit mit dem Spital des Kantons Jura. Dadurch sollen vor allem die Hausärzte entlastet werden und somit deren Arbeit an Attraktivität gewinnen. Bei rund 70000 Einwohnern (ca. 0,9\% der Schweizer Bevölkerung) auf einer Fläche von $838 \mathrm{~km}^{2}$ (2\% der Schweiz) ist die Grundversorgung auch geographisch schwierig. Aber umso interes- santer und dankbarer ist die Arzt-Patienten-Beziehung, da die Besonderheit der grossen Distanzen der Bevölkerung bewusst ist und einer kompetenten Vorortversorgung ein deutliches Gewicht zukommt.

\section{Aussichten}

Zurzeit ist die aktuelle Gesundheitspolitik völlig aus dem Ruder gelaufen und die Leidtragenden sind erneut die Randregionen. Es ist fast unmöglich, unseren jungen Kollegen die Grundversorgung anzubieten, wenn praktisch monatlich Hiobsbotschaften zu Abbau und Verschlechterung der Attraktivität des Berufs des Hausarztes sich in Ausmass und Intensität überschlagen. Die Frustration ist enorm und jede Bemühung, junge, gut ausgebildete Schweizer Ärzte anzuwerben, verläuft regelmässig im Sande.

Unser Anspruch ist keine Patentlösung aus Bern, sondern eine auf Föderalismus basierende Lösung der aktuellen Probleme ohne Seitenhiebe und Globalstrafen. Wir kennen unsere Bedürfnisse und Schwierigkeiten am besten und sind auch fähig und willig, Lösungen zu finden. Die Medikamentenabgabe zum Beispiel ist regional und im einzelnen geregelt und funktioniert perfekt. Dies zeigt sich auch in der Kostenstruktur im Gesundheitswesen des Kantons Jura. Die Zusammenarbeit zwischen dem Kanton und der Ärzteschaft funktioniert ausgezeichnet und wird beiderseits als Ergänzung empfunden. Zentrumsprobleme sollten nicht auf dem Rücken der Randregionen ausgetragen werden. Sobald wieder eine Gesundheitspolitik mit Rücksicht auf die regionalen Eigenheiten betrieben wird, können auch wir uns wieder mit vollem Elan auf unsere Haupttätigkeit konzentrieren: den Dienst am Patienten. Wir brauchen junge Ärzte, aber wo ist der Nachwuchs? Weshalb wird nicht mehr in die Nachwuchsförderung investiert für eine sichere Gesundheitsversorgung? 